\title{
Assessment of bone repair in animal models with suppressed osteoclast function by pretreatment of bisphosphonates
}

\author{
Yu Mori ${ }^{1}$ (D) Toshimi Aizawa ${ }^{1}$
}

Received: 22 June 2021 / Accepted: 7 July 2021 / Published online: 21 July 2021

(c) The Japanese Society Bone and Mineral Research 2021

\section{To the Editor}

We read with interest the article by Zhang et al. [1] titled "Long-term pretreatment with alendronate inhibits calvarial defect healing in an osteoporotic rat model". Long-term administration of bisphosphonates for the treatment of osteoporosis is associated with concerns of osteonecrosis of the jaw and atypical femur fractures, as well as impaired bone repair after fractures. Zhang et al. indicated the inhibitory effect of alendronate on the repair of bone defects in the skull of ovariectomized rats and the inability of parathyroid hormone $(\mathrm{PTH})$ administration to promote bone repair. The inhibition of osteogenesis in the study was reported to be related to the inhibition of the expression of Runt-related transcription factor 2 (RUNX2). On the other hand, we have reported impaired bone repair in a tibia fracture model in DNAX activating protein of $12 \mathrm{kDa}$ (DAP12)-deficient mice, which exhibit osteoclast suppression [2]. In DAP12deficient mice, the suppression of osteoclastgenesis and reduced inflammatory signaling resulted in impaired bone remodeling, including impaired cartilage resorption and bone formation in endochondral ossification. Previous study reported that low dose of zoledronate pretreatment did not affect the suppression of osteoblast proliferation and osteogenesis [3]. We speculated that the difference in the results of the two experiments may be due to the different doses and kinds of bisphosphonates. Although this study described the suppression of RUNX2 signaling, it did not describe the effect of the alendronate in differentiation of mesenchymal stem cells into osteoblasts or the induction of inflammation necessary for bone repair, which we hope will be clarified in future studies.

Yu Mori

yu-mori@med.tohoku.ac.jp

1 Department of Orthopaedic Surgery, Tohoku University Graduate School of Medicine, 1-1 Seiryo-machi, Aoba-ku, Sendai, Miyagi 980-8574, Japan
In the case of bone defect repair, there was no difference in the results of continued alendronate administration and PTH versus PTH alone, and bone repair was impaired in both cases compared to the control group. The results were interesting in that PTH did not contribute to bone repair. In contrast, it is not common to leave bone defect untreated, and bone grafting of artificial bone or autogenous bone is used for the treatment of bone defects in actual clinical practice. We have reported the effect of octacalcium phosphate artificial bone on bone repair in a rat ovariectomized osteoporosis model [4]. In the future, it might be worthwhile to study the inhibitory effect of long-term bisphosphonate administration on bone repair by bone grafting, comparing autogenous bone with artificial bone.

\section{References}

1. Zhang C, Zhu J, Jia J, Guan Z, Sun T, Zhang W, Yuan W, Wang H, Song C (2021) Long-term pretreatment with alendronate inhibits calvarial defect healing in an osteoporotic rat model. J Bone Miner Metab. https://doi.org/10.1007/s00774-021-01235-0

2. Kamimura M, Mori Y, Sugahara-Tobinai A, Takai T, Itoi E (2015) Impaired fracture healing caused by deficiency of the immunoreceptor adaptor protein DAP12. PLoS ONE. https://doi.org/10. 1371/journal.pone.0128210

3. Hu L, Wen Y, Xu J, Wu T, Zhang C, Wang J, Du J, Wang S (2017) Pretreatment with bisphosphonate enhances osteogenesis of bone marrow mesenchymal stem cells. Stem Cells Dev 26:123-132. https://doi.org/10.1089/scd.2016.0173

4. Baba K, Shiwaku Y, Hamai R, Mori Y, Anada T, Tsuchiya K, Oizumi I, Miyatake N, Itoi E, Suzuki O (2020) Chemical stabilitysensitive osteoconductive performance of octacalcium phosphate bone substitute in an ovariectomized rat tibia defect. ACS Appl Bio Mater 3:1444-1458. https://doi.org/10.1021/acsabm.9b01091

Publisher's Note Springer Nature remains neutral with regard to jurisdictional claims in published maps and institutional affiliations. 